\title{
Schmid Type Metaphyseal Chondrodysplasia in a Large Single Genetic Lineage
}

\author{
David Michaeli, Ravi Rajendra, Daniel C. Kim, Michael D. Goodlett, John R. Humphries, Prasit Nimityongskul
}

Department of Orthopaedic Surgery, University of South Alabama, Mobile, AL, USA

\section{Article Info}

\section{Article Notes}

Received: March 03, 2021

Accepted: May 08, 2021

\section{${ }^{*}$ Correspondence:}

Dr. David Michaeli, Department of Orthopaedic Surgery,

University of South Alabama, Mobile, AL, USA; Email:

dmichaeli@health.southalabama.edu.

${ }^{-} 2021$ Michaeli D. This article is distributed under the terms of the Creative Commons Attribution 4.0 International License.

\section{Keywords:}

Metaphyseal chondrodysplasia

Schmid Type

MCDS

pedigree

dwarfism

\section{Abstract}

Several subtypes of metaphyseal chondrodysplasia exist, of which the Schmid type is the most common. Characteristics include short limbed dwarfism, coxa vara, genu vara, and waddling gait. Skull, spine, and upper extremity involvement is minimal and often nonexistent. The primary defect involves a mutation affecting the metaphyseal portion of the growth plate, while the epiphysis is normal. A large single lineage family with metaphyseal chondrodysplasia, Schmid type (MCDS) was investigated. A genetic pedigree of 135 members of this family showed autosomal dominant inheritance between all 42 affected members. The large sample size allowed for the characterization of a broad range of features present in cases of MCDS. The majority of affected patients exhibited coxa vara with an average neck/shaft angle of 105 degrees. Despite coxa vara, premature osteoarthritis of the hip is not a feature of MCDS. Genu varum is the most prevalent knee disorder in this group, but greater than $30 \%$ of patients may exhibit genu valgum. This manuscript highlights MCDS background information, differential diagnoses, treatment options, and prognosis to aid in clinical decision-making.

\section{Introduction}

Metaphyseal chondrodysplasias (MCD) represent an array of conditions that present complex challenges for orthopaedic surgeons, as potentially, all long bones can be affected in unison. ${ }^{1}$ Radiographs reveal irregularities including splaying, flaring, cupping, and shortening of the tubular long bones with widened epiphyseal plates. Widespread coxa vara results in the quintessential waddling gait seen in patients (Figure 1). Sclerosis, and less commonly, vertebral platyspondyly and hand involvement may be present. In addition, joint pain has been reported in the past. $^{2}$

Schmid metaphyseal dyschondroplasia (MCDS) is an inherited metaphyseal disorder first described in $1949 .{ }^{3}$ Historically, MCDS was radiographically characterized by irregularities of the longbone metaphases with normal hand and vertebral development. ${ }^{4}$ Spine involvement was evident in scattered cases, but often resolved as patients aged. ${ }^{5}$ Today, MCDS is described as the development of progressive short stature with features usually absent at birth. Clinical characteristics manifest in early childhood with short limbs, genu varum, and waddling gait (Figure 2). Facial features and head size are typically normal, but adult height is several standard deviations below the mean, with a wide spectrum throughout affected individuals. Extraskeletal manifestations are absent, and affected individuals display normal intelligence. ${ }^{2}$

MCDS is caused by a mutation of the COL10A1 gene, which is transmitted in an autosomal dominant mode of inheritance. This 


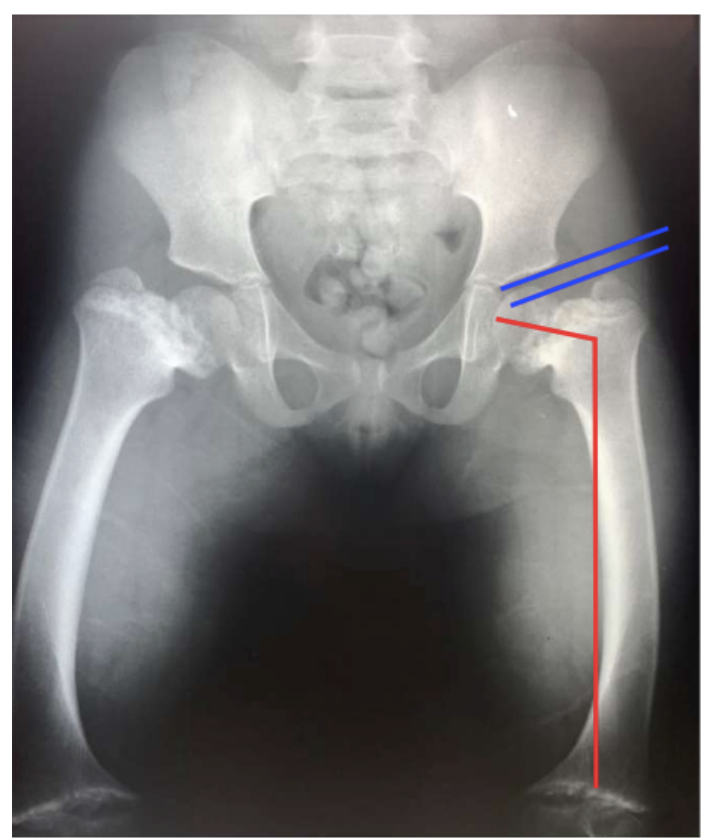

Figure 1. AP radiograph of pelvis and proximal femur in a known MCDS patient. The typical decreased neck-shaft angle (coxa vara) is seen (red). Furthermore, decreased articulo-trochanteric distance (distance between tangential lines about the superior acetabular articular surface and superior pole of the greater trochanter) accompanies the coxa vara deformity (blue). Splaying and flaring of the growth plates is evident.

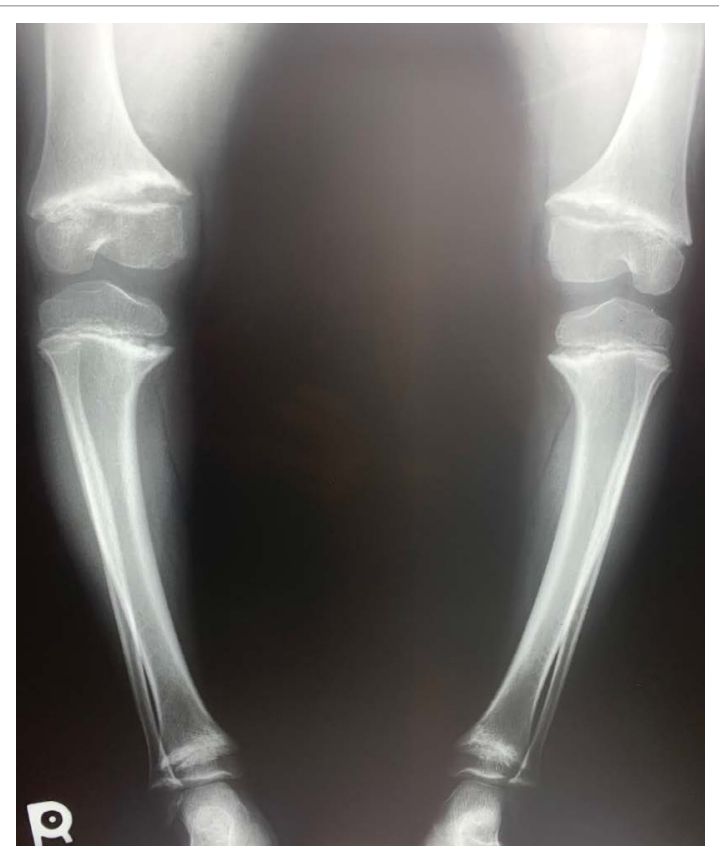

Figure 2. AP radiograph of knee and tibia in a known MCDS patient. Genu varus with irregularities in the growth plates can be seen bilaterally.

mutation quantitatively reduces collagen type $\mathrm{X}$ production, thereby reducing its presence at the hypertrophic zone adjacent to calcifying cartilage. ${ }^{1}$ Endochondral ossification, the process by which long bones elongate, is ultimately obscured. It is also the most common of the inherited metaphyseal chondrodysplasias, occurring at an estimated rate of three to six cases per million, which some current authors believe is a profound underestimation. ${ }^{6,7}$

Other, rarer metaphyseal chondrodysplasias with similar features to those of MCDS should be considered among the differential diagnoses. These include Jansen type, McKusick type (Cartilage-Hair Hypoplasia-CHH), and MCD with Malabsorption and Neutropenia (Shwachman Diamond Syndrome -SDS). CHH and SDS have additional multi-system involvement. Meticulous assessment of the patient, including judicious scrutiny of radiographs and analysis of complete laboratory markers, is crucial to avoid misdiagnosing these complex conditions with rickets. ${ }^{8}$

Regarded as the most severe subtype, Jansen metaphyseal chondrodysplasia (JMC) is a rare autosomal-dominant disease caused by activating mutations of PTHR1 resulting in inhibition of chondrocyte hypertrophic differentiation. ${ }^{6,9}$ Features of this disorder are more profound than those of MCDS and are detected at birth. ${ }^{6}$ In infancy, osteopenia, subperiosteal erosions, and hypercalcemia may cause confusion with hyperparathyroidism. ${ }^{10}$ Patients exhibit marked growth plate abnormalities, short stature and bowing of long bones, micrognathia, hypertelorism, and premature closure of cranial sutures. ${ }^{9}$

Cartilage-hair hypoplasia $(\mathrm{CHH})$ is a rare autosomal recessive metaphyseal chondrodysplasia caused by mutations of the untranslated $R M R P$ gene. It is characterized by short stature with a spectrum of skeletal abnormalities, fine sparse hair, and immune deficiency that can lead to recurrent infections. ${ }^{11}$ In the newborn, the first sign is bowing of the femora which is diagnostic, with metaphyseal changes only apparent after four months of age. $^{12}$

Similar to $\mathrm{CHH}$, Shwachman Diamond Syndrome affects multiple organ systems. A mutated SBDS gene on chromosome 7, normally involved in ribosome biogenesis and mitotic spindle stabilization, is disrupted, leading to the disease manifestation. ${ }^{13}$ Besides the immune, gastrointestinal, and cardiac features, SDS patients commonly exhibit skeletal abnormalities. Uniquely, these bony changes are due to failure of the columnar cartilage cells to hypertrophy. ${ }^{12}$ The primary defects involve abnormal development of growth plates, most frequently the femoral head. ${ }^{14}$ Other findings include rib cage abnormalities, metaphyseal dysostosis, osteopenia, and slipped capital femoral epiphysis (SCFE). ${ }^{15}$

Patients with metaphyseal dysmorphisms may present a challenging clinical diagnosis to healthcare providers. The differential diagnoses can be numerous, but special care must be taken to differentiate chondrodysplasias from rickets. In an attempt to reduce overall morbidity, evaluation 
of calcium and vitamin D is an essential component of initial management. Vitamin D deficiency is then subcategorized into severe deficiency $(<5 \mathrm{ng} / \mathrm{mL})$, moderate deficiency (5$15 \mathrm{ng} / \mathrm{mL})$, and insufficiency (16-20 ng/mL). ${ }^{16}$ Recognition of metaphyseal chondrodysplasia and differentiation from rickets is essential due to contrasting clinical treatments. While vitamin D supplementation is the remedy for rickets, it may precipitate toxicity in patients with MCDS. ${ }^{12,16,17}$

Orthopaedic treatment in MCDS is primarily confined to the lower extremities. Regarding gait, waddling is suggested to be caused by insufficient gluteal muscles. ${ }^{18}$ Patients have decreased articulo-trochanteric distance (ATD) resulting in decreased gluteal mechanical advantage. ${ }^{19}$ Usually, the entire femur shows a varus bow with the clinical appearance of genu varum. In some patients, varus alignment may improve spontaneously during childhood.

Some indications for surgical correction include significant coxa vara, a triangular fragment in the inferior femoral neck, and progressive deformity. Unfortunately, recurrence of deformity with longitudinal growth is common in many patients who have undergone surgical treatment. Guided growth procedures, such as hemiepiphysiodesis using eight-plates or stapling, may improve angular deformities in some children. ${ }^{20,21,22}$
Much of the research regarding MCDS occurs by studying index families, thus supporting the autosomal dominant transmission hypothesis. ${ }^{6}$ One such family has been identified by our institution, and our investigation is arguably one of the largest regarding this disease pattern in the literature.

\section{Materials And Methods}

A large, single family affected by this disorder had been noticed by our institution when the orthopaedic surgery department began rendering services to the state Children's Rehabilitation Service (previously Crippled Children's Clinic). Forty-two members of this family exhibited MCDS and underwent a thorough history, physical exam, as well as radiographic follow-up for a minimum of twenty years All affected members manifested radiographic signs of MCDS. There were no breaks in the hereditary link or skipped generations. There was no history of consanguinity in the family.

The following clinical measurements were obtained on each affected individual of the family: standing height, sitting height, arm span length, angulation of the knee, and hip range of motion (Table 1). Normal height was established using data from the National Health Survey

Table 1. Age, sitting/standing height, arm length, knee alignment, and radiographic characteristics of the 42 family members with MCDS evaluated in the study. Comments refer to relevant orthopaedic procedures undergone by patients prior to the study.

\begin{tabular}{|c|c|c|c|c|c|c|c|c|c|c|c|c|c|c|c|c|}
\hline \multirow{3}{*}{$\begin{array}{c}\text { No. } \\
1\end{array}$} & \multirow{3}{*}{$\begin{array}{c}\text { Name } \\
\text { Age/Sex }\end{array}$} & \multirow{3}{*}{\begin{tabular}{|c|}
$\begin{array}{c}\text { Height } \\
\text { Standing } \\
\text { (in/cm) }\end{array}$ \\
55 \\
139.7
\end{tabular}} & \multirow{3}{*}{\begin{tabular}{|l|}
$\mathbf{P}$ \\
0 \\
\end{tabular}} & \multirow{3}{*}{$\begin{array}{c}\begin{array}{c}\text { Sitting } \\
\text { Height } \\
\text { (in/cm) }\end{array} \\
32 \\
81.28 \\
\end{array}$} & \multirow{3}{*}{$\begin{array}{l}\mathbf{P} \\
0\end{array}$} & \multirow{3}{*}{\begin{tabular}{|c|}
$\begin{array}{c}\text { Arm Span } \\
\text { (in/cm) }\end{array}$ \\
56.5 \\
143.51 \\
\end{tabular}} & \multirow{3}{*}{$\begin{array}{c}\text { Knee } \\
\text { Alignment } \\
\text { Varus }\end{array}$} & \multirow{2}{*}{\multicolumn{2}{|c|}{$\begin{array}{c}\text { Hip } \\
\text { Internal } \\
\text { Rotation } \\
\mathbf{R} \quad \mathrm{L}\end{array}$}} & \multirow{2}{*}{\multicolumn{2}{|c|}{$\begin{array}{l}\text { Hip } \\
\text { External } \\
\text { Rotation } \\
\text { R L L }\end{array}$}} & \multicolumn{2}{|c|}{$\begin{array}{c}\text { Hip } \\
\text { Abduction }\end{array}$} & \multicolumn{2}{|c|}{$\begin{array}{c}\text { Neck Shaft } \\
\text { Angle }\end{array}$} & \multirow[t]{3}{*}{ Comments } \\
\hline & & & & & & & & & & & & $\mathbf{R}$ & $\mathbf{L}$ & $\mathbf{R}$ & $\mathbf{L}$ & \\
\hline & & & & & & & & 0 & 15 & 20 & 5 & 25 & 15 & 80 & 90 & \\
\hline 2 & $\begin{array}{c}\text { I.K. } \\
68+4 \mathrm{M}\end{array}$ & $\begin{array}{c}62.5 \\
158.75\end{array}$ & 0 & $\begin{array}{c}34 \\
86.36\end{array}$ & 0 & $\begin{array}{c}62 \\
157.48\end{array}$ & Valgus & 0 & 5 & 35 & 30 & 30 & 28 & 135 & 135 & \\
\hline 3 & $\begin{array}{c}\text { J.K. } \\
63+8 \mathrm{M}\end{array}$ & $\begin{array}{c}59 \\
149.86\end{array}$ & 0 & $\begin{array}{c}33 \\
83.82\end{array}$ & 0 & $\begin{array}{c}63 \\
150.02\end{array}$ & Varus & 13 & 9 & 40 & 30 & 30 & 35 & 100 & 120 & \\
\hline 4 & $\begin{array}{c}\text { C.K. } \\
48+1 \mathrm{M}\end{array}$ & $\begin{array}{c}59.5 \\
151.13\end{array}$ & 0 & $\begin{array}{c}32 \\
81.28\end{array}$ & 0 & $\begin{array}{c}60 \\
152.14\end{array}$ & Valgus & 5 & 23 & 35 & 27 & 30 & 30 & 110 & 110 & \\
\hline 5 & $\begin{array}{c}\text { M.K. } \\
60+10 \mathrm{M}\end{array}$ & $\begin{array}{c}64.5 \\
163.83\end{array}$ & 2 & $\begin{array}{c}34 \\
86.36\end{array}$ & 0 & $\begin{array}{c}72.5 \\
184.15\end{array}$ & Straight & 15 & 15 & 50 & 50 & 35 & 35 & 105 & 110 & \\
\hline 6 & $\begin{array}{c}\text { E.M. } \\
60+10 \mathrm{M}\end{array}$ & $\begin{array}{c}48.5 \\
123.2\end{array}$ & 0 & $\begin{array}{l}26.5 \\
67.3\end{array}$ & 0 & $\begin{array}{c}53 \\
134.6\end{array}$ & Valgus & 40 & 27 & 15 & 26 & 35 & 32 & 100 & 105 & \\
\hline 7 & $\begin{array}{c}\text { A.S. } \\
30+11 \mathrm{~F}\end{array}$ & $\begin{array}{c}60.75 \\
154.31\end{array}$ & 7 & $\begin{array}{c}33 \\
83.82\end{array}$ & 10 & $\begin{array}{c}53.25 \\
158.11\end{array}$ & Straight & 30 & 30 & 30 & 30 & 30 & 20 & 90 & 90 & \\
\hline 8 & $\begin{array}{c}\text { E.C. } \\
38+6 \mathrm{~F}\end{array}$ & $\begin{array}{c}58 \\
147.32\end{array}$ & 0 & $\begin{array}{c}31.5 \\
80.01\end{array}$ & 0 & $\begin{array}{c}60 \\
152.4\end{array}$ & Valgus & 27 & 27 & 30 & 40 & 30 & 20 & 95 & 95 & \\
\hline 9 & $\begin{array}{c}\text { A.R. } \\
29+7 \mathrm{~F}\end{array}$ & $\begin{array}{c}61.5 \\
156.21\end{array}$ & 10 & $\begin{array}{c}33 \\
83.82\end{array}$ & 10 & $\begin{array}{c}65 \\
165.1\end{array}$ & Valgus & 10 & 10 & 55 & 55 & 25 & 25 & 100 & 100 & \\
\hline 10 & $\begin{array}{c}\text { C.S. } \\
44+6 \mathrm{M}\end{array}$ & $\begin{array}{c}61 \\
155\end{array}$ & 0 & $\begin{array}{l}33.5 \\
85.1\end{array}$ & 0 & $\begin{array}{l}66.75 \\
169.6\end{array}$ & Valgus & 25 & 30 & 20 & 45 & 40 & 40 & 87 & 87 & \\
\hline 11 & $\begin{array}{c}\text { N.D. } \\
26+2 \mathrm{~F}\end{array}$ & $\begin{array}{c}51 \\
129.54\end{array}$ & 0 & $\begin{array}{c}30 \\
76.2\end{array}$ & 0 & $\begin{array}{c}55.5 \\
140.97\end{array}$ & Varus & 11 & 18 & 50 & 50 & 20 & 20 & 90 & 92 & \\
\hline 12 & $\begin{array}{c}\text { I.K. } \\
34+8 \mathrm{M}\end{array}$ & $\begin{array}{c}61.5 \\
156.21\end{array}$ & 0 & $\begin{array}{c}31 \\
78.74\end{array}$ & 0 & $\begin{array}{c}66 \\
167.64\end{array}$ & Straight & 15 & 15 & 35 & 35 & 35 & 35 & 115 & 115 & \\
\hline 13 & $\begin{array}{c}\text { N.M. } \\
11+11 \mathrm{M}\end{array}$ & $\begin{array}{c}57.5 \\
146.05\end{array}$ & 30 & $\begin{array}{c}28.5 \\
72.39\end{array}$ & 5 & $\begin{array}{c}60 \\
152.4\end{array}$ & Valgus & 8 & 8 & 82 & 82 & 35 & 35 & 100 & 100 & \\
\hline
\end{tabular}




\begin{tabular}{|c|c|c|c|c|c|c|c|c|c|c|c|c|c|c|c|c|}
\hline 14 & $\begin{array}{c}\text { N.M. } \\
27+1 \text { F }\end{array}$ & $\begin{array}{c}59 \\
149.86\end{array}$ & 0 & $\begin{array}{c}32 \\
81.28\end{array}$ & 0 & $\begin{array}{c}62 \\
157.48\end{array}$ & Valgus & 15 & 15 & 55 & 55 & 32 & 32 & 105 & 105 & \\
\hline 15 & $\begin{array}{c}\text { R.K. } \\
31+0 \mathrm{~F}\end{array}$ & $\begin{array}{c}56.74 \\
144.15\end{array}$ & 0 & $\begin{array}{c}32 \\
81.28\end{array}$ & 0 & $\begin{array}{c}61 \\
154.94\end{array}$ & Varus & 10 & 10 & 30 & 30 & 35 & 35 & 107 & 110 & \\
\hline 16 & $\begin{array}{c}\text { J.K. } \\
12+4 \mathrm{M}\end{array}$ & $\begin{array}{c}56 \\
142.24\end{array}$ & 10 & $\begin{array}{l}29.25 \\
72.29\end{array}$ & 10 & $\begin{array}{c}59 \\
149.86\end{array}$ & Valgus & 12 & 12 & 25 & 35 & 30 & 30 & 105 & 105 & \\
\hline 17 & $\begin{array}{c}\text { C.K. } \\
16+3 \mathrm{~F}\end{array}$ & $\begin{array}{c}60 \\
152.4\end{array}$ & 7 & $\begin{array}{c}31.5 \\
79.38\end{array}$ & 0 & $\begin{array}{c}61 \\
154.94\end{array}$ & $\begin{array}{l}\text { R Valgus } \\
\text { L Varus }\end{array}$ & 12 & 15 & 60 & 38 & 32 & 30 & 107 & 123 & \\
\hline 18 & $\begin{array}{c}\text { L.K. } \\
18+4 \mathrm{~F}\end{array}$ & $\begin{array}{c}59.5 \\
151.13\end{array}$ & 1 & $\begin{array}{c}31 \\
78.74\end{array}$ & 0 & $\begin{array}{c}64 \\
162.56\end{array}$ & Valgus & 35 & 35 & 32 & 27 & 28 & 25 & 105 & 105 & \\
\hline 19 & $\begin{array}{c}\text { R.K. } \\
41+3 \mathrm{M}\end{array}$ & $\begin{array}{c}64.5 \\
163.83\end{array}$ & 2 & $\begin{array}{c}33 \\
83.82\end{array}$ & 0 & $\begin{array}{c}67 \\
170.18\end{array}$ & Varus & 30 & 30 & 55 & 55 & 40 & 40 & 107 & 107 & \\
\hline 20 & $\begin{array}{c}\text { R.K. } \\
36+4 \mathrm{M}\end{array}$ & $\begin{array}{c}67 \\
170.18\end{array}$ & 15 & $\begin{array}{c}34 \\
86.36\end{array}$ & 0 & $\begin{array}{c}74 \\
187.96\end{array}$ & Varus & 35 & 35 & 30 & 30 & 43 & 43 & 112 & 112 & \\
\hline 21 & $\begin{array}{c}\text { T.P } \\
5+6 \mathrm{~F}\end{array}$ & $\begin{array}{c}34.5 \\
87.63\end{array}$ & 0 & $\begin{array}{c}23 \\
58.42\end{array}$ & 5 & $\begin{array}{c}40 \\
101.6\end{array}$ & Varus & 35 & 35 & 35 & 35 & 25 & 25 & 107 & 107 & \\
\hline 22 & $\begin{array}{l}\text { M.P. } \\
7+9 \mathrm{~F}\end{array}$ & $\begin{array}{c}45 \\
114.3\end{array}$ & 2 & $\begin{array}{c}26 \\
66.04\end{array}$ & 20 & $\begin{array}{c}47.5 \\
120.65\end{array}$ & Varus & 25 & 25 & 30 & 30 & 34 & 34 & 110 & 110 & \\
\hline 23 & $\begin{array}{l}\text { L.J. } \\
34+9 \mathrm{~F}\end{array}$ & $\begin{array}{c}59 \\
149.86\end{array}$ & 0 & $\begin{array}{c}34 \\
86.36\end{array}$ & 25 & $\begin{array}{c}61 \\
154.94\end{array}$ & $\begin{array}{l}\text { R Varus } \\
\text { L Straight }\end{array}$ & 30 & 25 & 30 & 25 & 25 & 25 & 90 & 88 & \\
\hline 24 & $\begin{array}{l}\text { K.M. } \\
10+6 \mathrm{~F}\end{array}$ & $\begin{array}{c}48.5 \\
123.2\end{array}$ & 0 & $\begin{array}{l}26 \\
66\end{array}$ & 0 & $\begin{array}{c}47.25 \\
120\end{array}$ & Valgus & 26 & 26 & 65 & 65 & 45 & 45 & 140 & 135 & $\begin{array}{l}\text { Bilateral Valgus Hip } \\
\text { Osteotomies }\end{array}$ \\
\hline 25 & $\begin{array}{l}\text { E.K. } \\
6+7 \mathrm{M}\end{array}$ & $\begin{array}{c}41.75 \\
106.05\end{array}$ & 0 & $\begin{array}{c}22 \\
55.88\end{array}$ & 0 & $\begin{array}{c}45.5 \\
115.57\end{array}$ & Varus & 12 & 12 & 35 & 35 & 40 & 40 & 110 & 110 & \\
\hline 26 & $\begin{array}{l}\text { R.K. } \\
2+4 \mathrm{M}\end{array}$ & $\begin{array}{c}33.5 \\
85.09\end{array}$ & 5 & $\begin{array}{c}20.5 \\
52.07\end{array}$ & 25 & $\begin{array}{l}33.75 \\
85.73\end{array}$ & Varus & 15 & 15 & 4 & 40 & 45 & 45 & 123 & 123 & \\
\hline 27 & $\begin{array}{l}\text { A.K. } \\
7+7 \mathrm{~F}\end{array}$ & $\begin{array}{c}42.75 \\
108.59\end{array}$ & 0 & $\begin{array}{c}25 \\
63.5\end{array}$ & 7 & $\begin{array}{c}46 \\
116.84\end{array}$ & Varus & 10 & 10 & 40 & 40 & 30 & 30 & 110 & 110 & \\
\hline 28 & $\begin{array}{l}\text { D.K. } \\
2+6 \mathrm{~F}\end{array}$ & $\begin{array}{c}31.5 \\
80.01\end{array}$ & 0 & $\begin{array}{l}18.75 \\
47.63\end{array}$ & 0 & $\begin{array}{c}32.5 \\
82.55\end{array}$ & Varus & 10 & 10 & 30 & 30 & 35 & 35 & 122 & 122 & \\
\hline 29 & $\begin{array}{l}\text { K.K. } \\
0+9 \mathrm{~F}\end{array}$ & $\begin{array}{l}24.75 \\
62.87\end{array}$ & 0 & $\begin{array}{c}17 \\
43.15\end{array}$ & 25 & $\begin{array}{c}26.5 \\
67.31\end{array}$ & Varus & 15 & 15 & 65 & 65 & 40 & 40 & 125 & 127 & \\
\hline 30 & $\begin{array}{l}\text { R.K. } \\
6+2 \mathrm{M}\end{array}$ & $\begin{array}{c}46.25 \\
117.48\end{array}$ & 45 & $\begin{array}{c}25 \\
63.5\end{array}$ & 25 & $\begin{array}{c}47.23 \\
120.02\end{array}$ & Valgus & 65 & 65 & 55 & 55 & 43 & 43 & 117 & 117 & \\
\hline 31 & $\begin{array}{l}\text { S.K. } \\
2+5 \mathrm{~F}\end{array}$ & $\begin{array}{c}35 \\
88.9\end{array}$ & 75 & $\begin{array}{c}21.5 \\
54.61\end{array}$ & 75 & $\begin{array}{c}34.5 \\
87.63\end{array}$ & Varus & 55 & 55 & 65 & 65 & 40 & 40 & 115 & 110 & \\
\hline 32 & $\begin{array}{c}\text { R.J. } \\
10+10 \mathrm{M}\end{array}$ & $\begin{array}{c}49.5 \\
125.73\end{array}$ & 50 & $\begin{array}{c}30 \\
76.2\end{array}$ & 50 & $\begin{array}{c}53 \\
134.62\end{array}$ & Varus & 20 & 0 & 15 & 25 & 20 & 15 & 95 & 110 & \\
\hline 33 & $\begin{array}{c}\text { B.C. } \\
17+5 \mathrm{~F}\end{array}$ & $\begin{array}{c}59 \\
149.86 \\
\end{array}$ & 10 & $\begin{array}{l}32.75 \\
83.19\end{array}$ & 10 & $\begin{array}{c}61 \\
154.94\end{array}$ & Varus & 10 & 20 & 45 & 45 & 35 & 35 & 87 & 90 & \\
\hline 34 & $\begin{array}{c}\text { F.C. } \\
19+6 \mathrm{M}\end{array}$ & $\begin{array}{c}59 \\
149.86\end{array}$ & 0 & $\begin{array}{c}34 \\
86.36\end{array}$ & 2 & $\begin{array}{c}60.5 \\
153.67\end{array}$ & Valgus & 10 & 10 & 42 & 42 & 35 & 30 & 80 & 90 & \\
\hline 35 & $\begin{array}{c}\text { L.S. } \\
17+3 \mathrm{M}\end{array}$ & $\begin{array}{c}62 \\
157.5\end{array}$ & 0 & $\begin{array}{l}37 \\
94\end{array}$ & 65 & $\begin{array}{c}67 \\
170.2\end{array}$ & Straight & 45 & 37 & 25 & 37 & 50 & 50 & 130 & 146 & $\begin{array}{l}\text { Bilateral Valgus Hip } \\
\text { Osteotomies } \\
\text { Tibial Osteotomies }\end{array}$ \\
\hline 36 & $\begin{array}{l}\text { R.S. } \\
17+8\end{array}$ & $\begin{array}{c}59 \\
150\end{array}$ & 0 & $\begin{array}{c}31.5 \\
80\end{array}$ & 0 & $\begin{array}{c}59 \\
150\end{array}$ & Varus & 30 & 45 & 30 & 30 & 30 & 42 & 105 & 105 & $\begin{array}{c}\text { Bilateral Valgus Hip } \\
\text { Osteotomies }\end{array}$ \\
\hline 37 & $\begin{array}{c}\text { E.S. } \\
15+8 \mathrm{M}\end{array}$ & $\begin{array}{c}59 \\
150\end{array}$ & 0 & $\begin{array}{l}32.5 \\
82.6\end{array}$ & 0 & $\begin{array}{c}62 \\
157.5\end{array}$ & $\begin{array}{l}R \text { Valgus } \\
\text { L Straight }\end{array}$ & 25 & 30 & 45 & 50 & 45 & 45 & 100 & 105 & $\begin{array}{c}\text { Bilateral Valgus Hip } \\
\text { Osteotomies }\end{array}$ \\
\hline 38 & $\begin{array}{c}\text { B.M. } \\
12+8 \mathrm{M}\end{array}$ & $\begin{array}{c}54.5 \\
138.43\end{array}$ & 2 & $\begin{array}{c}28.5 \\
72.39\end{array}$ & 1 & $\begin{array}{c}56 \\
142.24\end{array}$ & Varus & 10 & 10 & 45 & 45 & 35 & 35 & 102 & 102 & \\
\hline 39 & $\begin{array}{l}\text { C.M. } \\
12+1 \mathrm{~F}\end{array}$ & $\begin{array}{c}49.5 \\
125.73\end{array}$ & 0 & $\begin{array}{l}26.25 \\
67.31\end{array}$ & 0 & $\begin{array}{c}51.5 \\
130.81\end{array}$ & Varus & 15 & 15 & 50 & 50 & 30 & 30 & 105 & 105 & \\
\hline 40 & $\begin{array}{l}\text { R.K. } \\
6+4 \mathrm{M}\end{array}$ & $\begin{array}{c}41 \\
104.14\end{array}$ & 0 & $\begin{array}{c}23 \\
58.42\end{array}$ & 0 & $\begin{array}{c}43.5 \\
110.49\end{array}$ & Varus & 10 & 0 & 30 & 40 & 22 & 22 & 100 & 100 & \\
\hline 41 & $\begin{array}{c}\text { T.K. } \\
2+7 \mathrm{M}\end{array}$ & $\begin{array}{c}34 \\
86.36\end{array}$ & 5 & $\begin{array}{c}20 \\
50.8\end{array}$ & 5 & $\begin{array}{c}34 \\
86.36\end{array}$ & Varus & 15 & 15 & 40 & 40 & 32 & 32 & 125 & 124 & \\
\hline 42 & L.K. & $\begin{array}{c}31 \\
78.745\end{array}$ & 6 & $\begin{array}{c}17.5 \\
44.45\end{array}$ & 5 & $\begin{array}{c}30.5 \\
77.47\end{array}$ & Varus & 15 & 15 & 40 & 40 & 30 & 30 & 123 & 123 & \\
\hline
\end{tabular}


of height at skeletal maturity. A standard AP pelvis x-ray as well as AP $\mathrm{x}$-rays of both knees were taken in all affected individuals. Protrusio acetabuli was diagnosed using Kohler's line. Evidence of iliac wing hypoplasia was subjectively judged by the senior author.

\section{Results}

All individuals presented in our study manifest characteristic features of MCDS. The pedigree supports an autosomal dominant inheritance pattern (Figure 3).
Short stature is the main clinical presentation with a study average standing height of $145.6 \mathrm{~cm}$ and $156.9 \mathrm{~cm}$ in women and men, respectively. Overall average height for both males and females in skeletally mature MCD Schmid type is $151 \mathrm{~cm}$ (Table 2). The shortness of stature compared to the normal population is statistically significant $(\mathrm{p}<0.001)$. The average standing height of the 42 patients in this study is at the 5th percentile.

The difference in sitting height (Table 3) of the MCDS

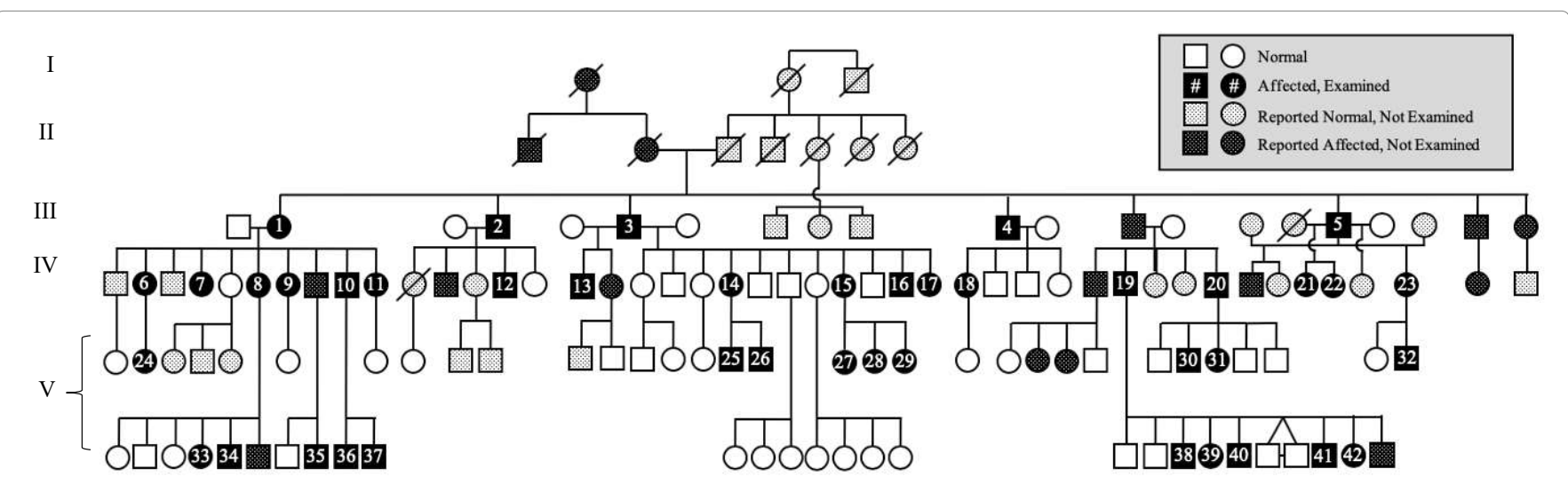

Figure 3. Pedigree representation of the MCDS cases within five generations of the study family. 135 members were investigated. 58 reportedly exhibited MCDS features, but only 42 participated in the study. Participants are numbered 1 through 42 . Completely shaded shapes $(n=42$; male $=21$, female $=21)$ are affected individuals who were examined. Unshaded are those who were normal and examined $(n=50 ;$ male $=31$, female $=15)$. Shaded shapes with white patterned dots represent individuals reportedly affected but not examined ( $n$ $=16$; male = 9, female $=7$ ). Unshaded shapes with black patterned dots represent individuals reported normal, but not examined $(n=27$; male $=12$, female $=15$ ). A line through a shape (shaded or not) represents a deceased individual.

Table 2. Average standing height in skeletally mature participants with MCD - Schmidt Type (Age 16 and above) compared to normal average standing height in United States. The difference in standing height between MCD and normal population is statistically significant at $P<0.001$.

\begin{tabular}{|c|c|c|c|c|}
\hline & Number of Patients & Avg. Standing Height inch $(\mathrm{cm})$ & Range inch (cm) & SD \\
\hline $\begin{array}{l}\text { MCD-Schmidt type: } \\
\text { Female, age > } 16\end{array}$ & 12 & $\begin{array}{c}57.33 \\
(145.63)\end{array}$ & $\begin{array}{c}48.5-61.5 \\
(123.2-156.21)\end{array}$ & $\begin{array}{c}3.97 \\
(10.1)\end{array}$ \\
\hline $\begin{array}{l}\text { Unaffected: } \\
\text { Female (US), age > } 16\end{array}$ & & $\begin{array}{c}63.86 \\
(162.2)\end{array}$ & & $\begin{array}{l}2.36 \\
(6.0)\end{array}$ \\
\hline $\begin{array}{l}\text { MCD-Schmidt type: } \\
\text { Male, age > } 16\end{array}$ & 11 & $\begin{array}{c}61.77 \\
(156.9)\end{array}$ & $\begin{array}{c}59.0-67.0 \\
(149.86-170.18)\end{array}$ & $\begin{array}{l}2.68 \\
(6.8)\end{array}$ \\
\hline $\begin{array}{l}\text { Unaffected: } \\
\text { Male (US), age >16 }\end{array}$ & & $\begin{array}{c}68.39 \\
(173.7)\end{array}$ & & $\begin{array}{c}2.61 \\
(6.64)\end{array}$ \\
\hline $\begin{array}{l}\text { MCD-Schmid type: } \\
\text { Female \& Male, age > } 16\end{array}$ & 23 & $\begin{array}{c}59.46 \\
(151.02)\end{array}$ & $\begin{array}{c}48.5-67.0 \\
(123.2-170.18)\end{array}$ & $\begin{array}{c}4.04 \\
(10.26)\end{array}$ \\
\hline
\end{tabular}

Table 3. Average sitting height in skeletally mature participants with MCD-Schmid type (age 16 and above) compared to normal average sitting height in the United States. The difference in sitting height between MCD and unaffected population is statistically significant at $P<0.001$.

\begin{tabular}{|l|c|c|c|c|}
\hline & No. & Avg. Sitting Height inch (cm) & Range inch (cm) & SD \\
\hline MCD-Schmidt type: & 12 & 31.6 & $26.5-34$ & 1.92 \\
\hline Female, age > 16 & & $(80.26)$ & $(67.31-86.36)$ & $1.38)$ \\
\hline Unaffected: & & 33.82 & & $(3.3)$ \\
\hline Female (US), age > 16 & 11 & $(85.9)$ & $31-37$ & $(78.74-94)$ \\
\hline MCD-Schmidt type: & & 33.4 & & 1.22 \\
Male, age > 16 & & $(84.84)$ & & $(3.1)$ \\
\hline Unaffected: & \multirow{2}{*}{23} & 36.02 & $26.5-37$ & 1.96 \\
Male (US), age >16 & & $(91.5)$ & $(67.3-94)$ & $(4.98)$ \\
\hline MCD-Schmid type: & & 32.44 & & \\
\hline Female \& Male, age $>16$ & & $(82.38)$ & & \\
\hline
\end{tabular}


patients compared to the normal population is also highly significant $(\mathrm{p}<0.001)$. The mean sitting height for the entire study group of patients is at the 9th percentile. In the skeletally mature group, the arm span is $7.25 \mathrm{~cm}$ longer than the standing height in females and $8.83 \mathrm{~cm}$ longer than the standing height in males, with the entire study group averaging a $6.48 \mathrm{~cm}$ difference in arm span and standing height (Tables $4-7$ ). On average, $90 \%$ and $79 \%$ of MCDS patients in the study were below the $10^{\text {th }}$ percentile in standing height and sitting height, respectively (Table 8)

Radiographically, all but seven study subjects demonstrated coxa vara with an average neck shaft angle of 105 degrees. Two additional individuals presented with

Table 4. Average sitting height as a percentage of average standing height of study participants.

\begin{tabular}{|l|c|c|c|}
\hline & No. & $\frac{\text { Sit Ht x 100\% }}{\text { Stand Ht }}$ & Range \\
\hline Female, age $>16$ & 12 & 55.15 & $52.1-58.8$ \\
\hline Male, age $>16$ & 11 & 54.06 & $50.4-59.7$ \\
\hline Female \& Male, age $>16$ & 23 & 54.63 & $50.4-59.7$ \\
\hline Female, age $<16$ & 9 & 59.56 & $53.5-68.6$ \\
\hline Male, age $<16$ & 10 & 55.27 & $49.6-61.2$ \\
\hline Female and male, age $<16$ & 19 & 57.3 & 4.87 \\
\hline Entire series & 42 & 55.84 & $49.6-68.6$ \\
\hline
\end{tabular}

Table 5. Average arm span in skeletally mature participants (age 16 and above) with MCDS.

\begin{tabular}{|l|c|c|c|c|}
\hline & Number of Patients & Avg. Standing Height inch (cm) & nge inch (cm) & SD inch (cm) \\
\hline MCD-Schmidt type: & 12 & 60.19 & $53-64$ & 3.51 \\
Female, age > 16 & & $(152.88)$ & $(134.6-162.56)$ & $(8.92)$ \\
\hline MCD-Schmidt type: & 11 & 65.25 & $59-72.5$ & 4.93 \\
Male, age > 16 & \multirow{2}{*}{23} & $(165.68)$ & $53-72.5$ & $(12.52)$ \\
\hline MCD-Schmid type: & & 62.61 & $(134.6-184.15)$ & 4.89 \\
Female \& Male, age $>16$ & & $(159.03)$ & $(12.42)$ \\
\hline
\end{tabular}

Table 6. Difference in length between arm span and standing height in study participants. (Normal $=<3.5 \mathrm{~cm}$ ). On average, participants' arm span is greater than standing height, suggesting greater involvement in the lower extremities.

\begin{tabular}{|c|c|c|c|c|}
\hline & No. & Avg arm span - standing height inches $(\mathrm{cm})$ & Range inches (cm) & SD. \\
\hline Female, age $>16$ & 12 & $\begin{array}{c}2.85 \\
(7.25)\end{array}$ & $\begin{array}{c}1-4.5 \\
(2.54-11.43)\end{array}$ & $\begin{array}{c}1.34 \\
(3.41)\end{array}$ \\
\hline Male, age $>16$ & 11 & $\begin{array}{c}3.48 \\
(8.83)\end{array}$ & $\begin{array}{c}-0.5-8.0 \\
(-1.27-20.32)\end{array}$ & $\begin{array}{c}2.89 \\
(7.33)\end{array}$ \\
\hline Female \& Male, age $>16$ & 23 & $\begin{array}{l}3.14 \\
(8.0)\end{array}$ & $\begin{array}{c}-0.5-8.0 \\
(-1.27-20.32)\end{array}$ & $\begin{array}{c}2.19 \\
(5.56)\end{array}$ \\
\hline Female, age $<16$ & 9 & $\begin{array}{c}1.64 \\
(4.16)\end{array}$ & $\begin{array}{c}-1.26-5.2 \\
(-3.2-13.97)\end{array}$ & $\begin{array}{c}2.03 \\
(5.16)\end{array}$ \\
\hline Male, age $<16$ & 10 & $\begin{array}{c}2.1 \\
(5.32)\end{array}$ & $\begin{array}{c}0-3.75 \\
(0-9.52)\end{array}$ & $\begin{array}{c}1.26 \\
(3.21)\end{array}$ \\
\hline Female and male, age $<16$ & 19 & $\begin{array}{c}1.88 \\
(4.77)\end{array}$ & $\begin{array}{c}-1.26-5.5 \\
(-3.2-13.97)\end{array}$ & $\begin{array}{c}1.66 \\
(4.23)\end{array}$ \\
\hline Entire series & 42 & $\begin{array}{c}2.55 \\
(6.48)\end{array}$ & $\begin{array}{c}-1.26-8.0 \\
(-3.2-20.32)\end{array}$ & $\begin{array}{c}2.08 \\
(5.28)\end{array}$ \\
\hline
\end{tabular}

Table 7. Arm span to standing height ratio in study participants (Normal $1.00-1.03$ ). With lower extremities affected more than upper extremities, the arm span to standing height ratio is increased to a value greater than the normal mean.

\begin{tabular}{|l|c|c|c|c|}
\hline & No. & Arm Span/Standing Height Ratio & Range & S.D. \\
\hline Female, age $>16$ & 12 & 1.05 & $1.02-1.09$ & 0.027 \\
\hline Male, age $>16$ & 11 & 1.05 & $0.99-1.12$ & 0.04 \\
\hline Female \& Male, age $>16$ & 23 & 1.05 & $0.99-1.10$ & 0.035 \\
\hline Female, age $<16$ & 9 & 1.04 & $0.97-1.16$ & 0.06 \\
\hline Male, age $<16$ & 10 & 1.04 & $1.00-1.09$ & 0.03 \\
\hline Female and male, age $<16$ & 19 & 1.04 & $0.97-1.16$ & 0.04 \\
\hline Entire series & 42 & 1.05 & $0.97-1.16$ & 0.04 \\
\hline
\end{tabular}


Table 8. Proportion of study participants whose standing height and sitting height is at or below $5^{\text {th }}$ and $10^{\text {th }}$ percentile..$^{25}$

\begin{tabular}{|c|c|c|}
\hline & No./Total & Percent \\
\hline Participants with Standing Height at or Below 5th Percentile & $26 / 42$ & $62 \%$ \\
\hline Participants with Standing Height at or Below 10th Percentile & $38 / 42$ & $90 \%$ \\
\hline Participants with Siting Height at or Below 5th Percentile & $23 / 42$ & $55 \%$ \\
\hline Participants with Sitting Height at or Below 10th Percentile & $33 / 42$ & $79 \%$ \\
\hline
\end{tabular}

neck shaft angles $>120$, but they had previously undergone valgus osteotomies. The normal femoral neck shaft angle is between 125-135 degrees, and varus is defined here as $\leq 120$. Follow-up radiographs of the hips showed no evidence of premature osteoarthritis of the hips in this condition. In this study, $60 \%$ of patients exhibited iliac hypoplasia. No evidence of protrusio acetabuli was seen in any of the radiographs.

In studies on MCDS, knee alignment is reported to be in varus. In this series of 42 patients, approximately $50 \%$ of the patients demonstrated genu varum, approximately $33 \%$ demonstrated genu valgum, and approximately $10 \%$ of our population had straight knees.

Patients in this series exhibited normal life spans with no evidence of detectable intellectual deficits. No other congenital abnormalities of the musculoskeletal system were seen caudal to the knees in these patients.

\section{Discussion}

In this study, we describe the largest reported series of individuals of a single lineage who demonstrate features consistent with MCDS. Short stature and proximal femur involvement, specifically coxa vara, are defining characteristics of this condition. ${ }^{23}$ Eighty-three percent of the patients in our study exhibited these two main clinical features. Autosomal dominant inheritance of MCDS with no skipped generations was noted for the 42 affected members in this large single lineage study. The mean standing height of MCDS females and males in our study was $145 \mathrm{~cm}$ and $156 \mathrm{~cm}$, respectively. In comparison, the mean height of normal U.S. females and males is $162 \mathrm{~cm}$ and $172 \mathrm{~cm}$, respectively. These results are both statistically significant compared to the normal population $(\mathrm{p}<0.001)$ and consistent with Schmid's original sample describing overall average standing height for MCDS individuals as $150 \mathrm{~cm}$. In addition, our findings confirm that the short stature is due to dysplasia of the lower extremities rather than that of the axial skeleton, with an average sitting to standing height ratio of 0.55 , approximately two standard deviations higher than normal individuals. ${ }^{24}$ Moreover, our findings agree with previous reports in the literature of minimal skull, spine, or trunk involvement. ${ }^{6}$

Our study confirms previous radiological findings related to proximal femur deformity with $83 \%$ of our patients presenting with coxa vara and an average neck shaft angle of 105 degrees. All study subjects declined elective surgery to correct bony alignment, gait deformity, and muscular insufficiency. This is likely because the study population is from a single lineage with individuals who are aware of the natural progression of MCDS in their family. Remarkably, despite this deformity, the vast majority of our study population remained clinically functional with very little limitation of activities. Premature osteoarthritis of the hips was not a notable feature in our study population, and only one patient in his 60s showed evidence of osteoarthritis. Most of the study population, however, did exhibit waddling gait due to weakness of the gluteus medius in the setting of coxa vara.

Due to the breadth of our patient population, we were able to elucidate more patterns associated with MCDS. Previous accounts described predominantly genu varum presentations estimating greater than $60 \%$ of patients with MCDS have genu varum. ${ }^{2}$ However, while our study population consistently demonstrated coxa vara deformity proximally, variance in deformity occurred distally at the knee. Of the 42 patients, only 50\% demonstrated genu varum, notably less than estimates in current literature. Approximately $33 \%$ of the study population demonstrated genu valgum and $10 \%$ had straight knees. Our results suggest that diversity of deformity can occur at the knee joint and must be considered by the orthopaedic community in diagnosing and treating MCDS.

\section{Conclusion}

This is the largest reported series of a single lineage MCDS. Autosomal dominant mode of inheritance is confirmed in our pedigree. Subischial involvement in the lower extremities predominates compared to involvement of the trunk or spine. The skull and upper extremities are relatively normal in these patients. Coxa vara is a consistent finding occurring in the majority of our patients, but this confers minimal lifestyle disability with no evidence for premature osteoarthritis of the hips. The previously established knee deformity in this condition is varus, but our series revealed a third of patients with valgus knees. The long-term follow-up reveals that these patients exhibit a normal life span in the setting of these findings.

\section{References}

1. Woelfle JV, Brenner RE, Zabel B, Reichel H, Nelitz M. Schmid-type metaphyseal chondrodysplasia as the result of a collagen type $\mathrm{X}$ defect due to a novel COL10A1 nonsense mutation: A case report of a novel COL10A1 mutation. J Orthop Sci. 2011;16(2):245-249. 
2. Richmond CM, Savarirayan R. Schmid Metaphyseal Chondrodysplasia In: Adam MP, Ardinger HH, Pagon RA, et al, eds. GeneReviews®. Seattle: University of Washington; 2019.

3. Schmid F. Beitrag zur Dysostosis enchondralis metaphysarea. Monats Kinderheilkd. 1949(97):393-397.

4. Lachman RS, Rimoin DL, Spranger J. Metaphyseal chondrodysplasia, Schmid type. Clinical and radiographic delineation with a review of the literature. Pediatr Radiol. 1988;18(2):93-102.

5. Savarirayan R, Cormier-Daire V, Lachman RS, Rimoin DL. Schmid type metaphyseal chondrodysplasia: a spondylometaphyseal dysplasia identical to the "Japanese" type. Pediatr Radiol. 2000;30(7):460-463.

6. Al Kaissi A, Ghachem MB, Nabil NM, et al. Schmid's Type of Metaphyseal Chondrodysplasia: Diagnosis and Management. Orthop Surg. 2018;10(3):241-246.

7. Gokhale S, Mehta S. Schmid type metaphyseal chondrodysplasia. Indian Pediatr. 2005;42(12):1252.

8. Frame B, Poznanski AK. Conditions that may be confused with rickets. In: DeLuca HF, Anast CS, eds. Pediatric Diseases Related to Calcium. New York, NY: Elsevier; 1980:269-289.

9. Saito $\mathrm{H}$, Noda $\mathrm{H}$, Gatault $\mathrm{P}$, et al. Progression of Mineral Ion Abnormalities in Patients With Jansen Metaphyseal Chondrodysplasia. J Clin Endocrinol Metab. 2018;103(7):2660-2669.

10. Kozlowski K, Campbell JB, Azouz ME, Sprague P. Metaphyseal chondrodysplasia, type Jansen. Australas Radiol. 1999;43(4):544-547.

11. Sathishkumar D, Gach JE, Ogboli M, et al. Cartilage hair hypoplasia with cutaneous lymphomatoid granulomatosis. Clin Exp Dermatol. 2018;43(6):713-717.

12. Spranger JW. Metaphyseal chondrodysplasia. Postgrad Med J. 1977;53(622):480-487.

13. Boocock GR, Morrison JA, Popovic M, et al. Mutations in SBDS are associated with Shwachman-Diamond syndrome. Nat Genet. 2003;33(1):97-101.

14. Topa A, Tulinius M, Oldfors A, Hedberg-Oldfors C. Novel myopathy in a newborn with Shwachman-Diamond syndrome and review of neonatal presentation. Am J Med Genet A. 2016;170A(5):1155-1164.

15. Ginzberg H, Shin J, Ellis L, et al. Shwachman syndrome: phenotypic manifestations of sibling sets and isolated cases in a large patient cohort are similar. J Pediatr. 1999;135(1):81-88.

16. Lee JY, So TY, Thackray J. A review on vitamin d deficiency treatment in pediatric patients. J Pediatr Pharmacol Ther. 2013;18(4):277-291.

17. Munns CF, Shaw N, Kiely M, et al. Global Consensus Recommendations on Prevention and Management of Nutritional Rickets. J Clin Endocrinol Metab. 2016;101(2):394-415.

18. Tauber C, Ganel A, Horoszowski H, Farine I. Distal transfer of the greater trochanter in cox vara. Acta Orthop Scand. 1980;51(4):661-666.

19. Yoon PW, Shin YH, Yoo JJ, Yoon KS, Kim HJ. Progression of a fracture site impaction as a prognostic indicator of impacted femoral neck fracture treated with multiple pinning. Clin Orthop Surg. 2012;4(1):66-71.

20. Rosenbloom AL, Smith DW. The Natural History Of Metaphyseal Dysostosis. J Pediatr. 1965;66:857-868.

21. Bassett GS. Orthopaedic aspects of skeletal dysplasias. Instr Course Lect. 1990;39:381-387.

22. Stevens PM, Klatt JB. Guided growth for pathological physes: radiographic improvement during realignment. J Pediatr Orthop. 2008;28(6):632-639.

23. Mäkitie O, Susic M, Ward L, Barclay C, Glorieux FH, Cole WG. Schmid type of metaphyseal chondrodysplasia and COL10A1 mutations-findings in 10 patients. Am J Med Genet A. 2005;137A(3):241-248.

24. Fredriks AM, van Buuren S, van Heel WJ, Dijkman-Neerincx RH, Verloove-Vanhorick SP, Wit JM. Nationwide age references for sitting height, leg length, and sitting height/height ratio, and their diagnostic value for disproportionate growth disorders. Arch Dis Child. 2005;90(8):807-812.

25. Hamill PV, Drizd TA, Johnson CL, Reed RB, Roche AF. NCHS growth curves for children birth-18 years. United States. Vital Health Stat 11. 1977(165):i-iv, 1-74. 